Proc. Indian Acad. Sci. (Math. Sci.) Vol. 113, No. 3, August 2003, pp. 213-221.

Printed in India

\title{
Analogues of Euler and Poisson summation formulae
}

\author{
VIVEK V RANE \\ Department of Mathematics, The Institute of Science, 15, Madam Cama Road, \\ Mumbai 400 032, India
}

MS received 2 February 2002; revised 20 January 2003

\begin{abstract}
Euler-Maclaurin and Poisson analogues of the summations $\sum_{a<n \leq b} \chi(n) f(n)$ $\sum_{a<n \leq b} d(n) f(n), \sum_{a<n \leq b} d(n) \chi(n) f(n)$ have been obtained in a unified manner, where $(\chi(n))$ is a periodic complex sequence; $d(n)$ is the divisor function and $f(x)$ is a sufficiently smooth function on $[a, b]$. We also state a generalised Abel's summation formula, generalised Euler's summation formula and Euler's summation formula in several variables.
\end{abstract}

Keywords. Abel's summation formula; Euler summation formula; Euler-Maclaurin summation formula; Poisson's summation formula; Fourier series.

\section{Introduction}

Voronoi [4] conjectured that if $(c(n))$ is a given arithmetical function and if $f$ is a continuous function on an interval $[a, b]$ with only finite number of maxima and minima there, then there exist analytic functions $\alpha(x)$ and $\delta(x)$, depending only upon $c(n)$ (and not upon $f(x)$ ) such that

$$
\sum_{a \leq n \leq b}{ }^{\prime} c(n) f(n)=\int_{a}^{b} f(x) \delta(x) \mathrm{d} x+\sum_{n=1}^{\infty} c(n) \int_{a}^{b} f(x) \alpha(n x) \mathrm{d} x .
$$

The prime on the summation sign $\sum_{a \leq n \leq b}^{\prime} c(n) f(n)$ means that if $n=a$ or $n=b$, only $(1 / 2) c(a) f(a)$ or $(1 / 2) c(b) f(b)$ respectively is counted. In the special case $c(n)=d(n)$, where $d(n)=$ the number of divisors of $n$, Voronoi obtained the formula

$$
\sum_{a \leq n \leq b}{ }^{\prime} d(n) f(n)=\int_{a}^{b}(\log x+2 \gamma) f(x) \mathrm{d} x+\sum_{n=1}^{\infty} d(n) \int_{a}^{b} f(x) \alpha(n x) \mathrm{d} x,
$$

with $\alpha(x)=4 K_{0}(4 \pi \sqrt{x})-2 \pi Y_{0}(4 \pi \sqrt{x})$, where $K_{0}$ and $Y_{0}$ are the well-known Bessel functions and $\gamma$ is the Euler's constant. Obviously, Voronoi's summation formula is a generalisation of Poisson's summation formula. Berndt [1] and Berndt and Schoenfeld [2] have given the Euler-Maclaurin and the Poisson analogues of the summation $\sum_{a<n \leq b} \chi(n) f(n)$, where $(\chi(n))$ is a periodic sequence of complex numbers.

The object of this paper is as follows:

(1) To give the analogues of the Euler-Maclaurin's summation formula of $\sum_{a<n<b} \chi(n) f(n)$, $\sum_{a<n \leq b} d(n) f(n)$ and $\sum_{a<n \leq b} d(n) \chi(n) f(n)$, where $f(x)$ is a sufficiently smooth function on the interval $[a, b] ; d(n)$ is the divisor function and $(\chi(n))$ is a periodic sequence of complex numbers of period $k$ (see Theorem 1 ). 
(2) To give the analogues of the Poisson's summation formula for $\sum_{a<n \leq b} \chi(n) f(n)$, $\sum_{a<n \leq b} d(n) f(n)$ and $\sum_{a<n \leq b} d(n) \chi(n) f(n)$ (see Theorem 2). These will be accomplished virtually effortlessly without the use of complex contour integration. Incidentally in particular, choosing $\chi(n)$ to be the constant sequence $(1)_{n}$ so that $k=1$, we can obtain Euler-Maclaurin and Poisson summation formulae for $\sum_{a<n \leq b} f(n)$ from the corresponding results for $\sum_{a<n \leq b} \chi(n) f(n)$.

Next, we introduce our notation and state our theorems.

Notation. In what follows, the summation $\sum_{n=-\infty}^{\infty}$ or $\sum_{n}$ will always mean the summation in the sense $\lim _{N \rightarrow \infty} \sum_{|n| \leq N}$; and $\sum_{n=-\infty}^{\prime \infty}$ or $\sum_{n}^{\prime}$ shall denote exclusion of the term corresponding to $n=0$. For $x$ real, $[x]$ denotes the integral part of $x$. We shall write $\psi(x)=x-[x]-(1 / 2)$. Thus for non-integer $x$, we have $\psi(x)=-\sum^{\prime}{ }_{n}\left(\mathrm{e}^{2 \pi i n x} / 2 \pi i n\right)$. We shall call a Riemann-integrable function $f$ on an interval $[a, b]$ of the real line, a good function on $[a, b]$, if it admits the interchange of $\sum$ and $\int$ in the Riemann-Stieltjes integral $\int_{a}^{b} \psi(\alpha x+\beta) \mathrm{d} f(x)$, i.e.,

$$
-\int_{a}^{b} \sum_{n=-\infty}^{\infty}, \frac{\mathrm{e}^{2 \pi i n(\alpha x+\beta)}}{2 \pi i n} \mathrm{~d} f(x)=-\sum_{n=-\infty}^{\infty}{ }^{\prime} \int_{a}^{b} \frac{\mathrm{e}^{2 \pi i n(\alpha x+\beta)}}{2 \pi i n} \mathrm{~d} f(x),
$$

where $\alpha, \beta$ are some real constants with $0<\alpha \leq 1$. (If $f$ is differentiable with its derivative finite and integrable, whether in the Riemann sense or in the Lebesgue sense, on $[a, b]$, then $f$ is good on $[a, b]$. Also, if $f$ is a function of bounded variation, then $f$ is good.)

For an integer $r \geq 0$, we shall write

$$
\psi_{r}(x)=-\sum_{n}^{\prime} \frac{\mathrm{e}^{2 \pi i n x}}{(2 \pi i n)^{r+1}}=\frac{B_{r+1}(x)}{(r+1) !}
$$

where $B_{r}(x)$ is the Bernoulli polynomial of degree $r$ and $(r+1)$ ! denotes $(r+1)$ factorial. Note that $\psi_{0}(x)=\psi(x)$ and $(\mathrm{d} / \mathrm{d} x) \psi_{r}(x)=\psi_{r-1}(x)$ for $r \geq 1$.

Next, we state our theorems.

Theorem 1. Let the function $f$ have continuous derivatives up to $(R+1)$ th order on $[a, b]$.

(I) Let $(\chi(n))$ be a complex sequence with period $k$ and let $d(n)$ be the divisor function. Then, we have

$$
\begin{aligned}
\sum_{a<n \leq b} \chi(n) f(n)= & \frac{1}{k} \sum_{l=1}^{k} \chi(l) \int_{a}^{b} f(u) \mathrm{d} u \\
& -\sum_{r=0}^{R}(-k)^{r}\left\{f^{(r)}(b) \sum_{n}^{\prime} \tau(\chi,-n) \frac{\mathrm{e}^{2 \pi i n b / k}}{(2 \pi i n)^{r+1}}\right. \\
& \left.-f^{(r)}(a) \sum_{n}^{\prime} \tau(\chi,-n) \frac{\mathrm{e}^{2 \pi i n a / k}}{(2 \pi i n)^{r+1}}\right\} \\
& +(-k)^{R} \sum_{n}^{\prime} \frac{\tau(\chi,-n)}{(2 \pi i n)^{R+1}} \int_{a}^{b} f^{(R+1)}(u) \mathrm{e}^{2 \pi i n u / k} \mathrm{~d} u
\end{aligned}
$$

where $\tau(\chi, n)=\sum_{l=1}^{k} \chi(l) \mathrm{e}^{2 \pi i n l / k}$. 
(II) We have

$$
\begin{aligned}
& \sum_{a<n \leq b} d(n) f(n) \\
& =\int_{a}^{b} f(u) \mathrm{d}(u)\left(\sum_{m \leq b} \frac{1}{m}\right) \\
& \quad+\sum_{r=0}^{R}(-1)^{(r+1)}\left\{f^{(r)}(b) \sum_{n}^{\prime} \frac{1}{(2 \pi i n)^{r+1}}\left(\sum_{m \leq b} m^{r} \mathrm{e}^{2 \pi i n b / m}\right)\right. \\
& \left.\quad-f^{(r)}(a) \sum_{n}^{\prime} \frac{1}{(2 \pi i n)^{r+1}}\left(\sum_{m \leq b} m^{r} \mathrm{e}^{2 \pi i n a / m}\right)\right\} \\
& \quad+(-1)^{R} \sum_{n}^{\prime} \frac{1}{(2 \pi i n)^{R+1}} \int_{a}^{b} f^{(R+1)}(u)\left(\sum_{m \leq b} m^{R} \mathrm{e}^{2 \pi i n u / m}\right) \mathrm{d} u .
\end{aligned}
$$

(III) We have

$$
\begin{aligned}
\sum_{a<n \leq b} \chi(n) d(n) f(n)= & \frac{1}{k} \sum_{r_{1}=1}^{k} \sum_{r_{2}=1}^{k} \chi\left(r_{1} r_{2}\right)\left(\sum_{\substack{m \leq b \\
m \equiv r_{1}(\bmod k)}} \frac{1}{m} \int_{a}^{b} f(u) \mathrm{d} u\right) \\
& +\sum_{r=0}^{R}(-1)^{r+1} k^{r} \sum_{r_{1}=1}^{k} \sum_{r_{2}=1}^{k} \chi\left(r_{1} r_{2}\right) \\
& \times\left\{f^{(r)}(b) \sum_{n}^{\prime} \frac{\mathrm{e}^{-2 \pi i n r_{2} / k}}{(2 \pi i n)^{r+1}} \cdot \sum_{\substack{m \leq b \\
m \equiv r_{1}(\bmod k)}} \mathrm{e}^{2 \pi i n b / m k}\right. \\
& +(-k)^{R} \sum_{r_{1}=1}^{k} \sum_{r_{2}=1}^{k} \chi\left(r_{1}(a) \sum_{n}^{\prime} \frac{\mathrm{e}^{-2 \pi i n r_{2} / k}}{(2 \pi i n)^{r+1}} \cdot \sum_{n}^{\prime} \sum_{\substack{m \leq b \\
m \equiv r_{1}(\bmod k)}} \mathrm{e}^{-2 \pi i n r_{2} / k} \int_{a}^{b} f^{(R+1)}(u)\right. \\
& \left(\begin{array}{c}
\left.\sum_{m \leq b} \mathrm{e}^{2 \pi i n u / m k}\right) \\
\mathrm{d} u .
\end{array}\right.
\end{aligned}
$$

Theorem 2. Let a function $f$ be good on the interval $[a, b]$. Let $(\chi(n))$ be a complex sequence of period $k$ and let $d(n)$ be the divisor function. Then, we have

$$
\sum_{a<n \leq b} \chi(n) f(n)=\frac{1}{k} \sum_{n=-\infty}^{\infty} \tau(\chi, n) \cdot \int_{a}^{b} f(u) \mathrm{e}^{-2 \pi i n u / k} \mathrm{~d} u,
$$

where $\tau(\chi, n)=\sum_{l=1}^{k} \chi(l) \mathrm{e}^{2 \pi i n l / k}$.

(II) We have

$$
\sum_{a<n \leq b} d(n) f(n)=\sum_{n=-\infty}^{\infty} \int_{a}^{b} f(u)\left(\sum_{m \leq b} \frac{1}{m} \mathrm{e}^{2 \pi i n u / m}\right) \mathrm{d} u .
$$


(III)

$$
\begin{aligned}
\sum_{a<n \leq b} \chi(n) d(n) f(n) \\
=\sum_{r_{1}=1}^{k} \sum_{r_{2}=1}^{k} \chi\left(r_{1} r_{2}\right) \sum_{n=-\infty}^{\infty} \mathrm{e}^{-2 \pi i n r_{2} / k} \\
\quad \times \int_{a}^{b} \mathrm{~d} u f(u)\left(\sum_{\substack{m \leq b \\
m \equiv r_{1}(\bmod k)}} \frac{1}{m} \mathrm{e}^{2 \pi i n u / m k}\right) \\
\quad+\frac{1}{k} \sum_{r_{1}=1}^{k} \sum_{r_{2}=1}^{k} \chi\left(r_{1} r_{2}\right)\left(\int_{a}^{b} f(u) \mathrm{d} u\right)\left(\sum_{\substack{m \leq b \\
m \equiv r_{1}(\bmod k)}} \frac{1}{m}\right) .
\end{aligned}
$$

Berndt [1] and Berndt and Schoenfeld [2] have given the Euler-Maclaurin and the Poisson analogues for the summation $\sum_{a<n<b} \chi(n) f(n)$ by different methods. Their results are similar to our results for $\sum_{a<n \leq b} \chi(n) f(n)$. Jutila [3] obtained transformation formulae in analytic number theory, where he deals with the summations $\sum_{n} d(n) f(n)$ and $\sum_{n} a(n) f(n) ; a(n)$ being the $n$th Fourier coefficient of a cusp form. We can also obtain the Euler-Maclaurin and the Poisson analogues of $\sum_{n} a(n) f(n)$, using our approach. More generally, we can deal with the summation $\sum_{n} r(n) f(n)$, where $r(n)$ is the Fourier coefficient of a periodic integrable function $g(x)$ of period 1 . Thus writing $r(x)=\int_{0}^{1} g(u) \mathrm{e}^{-2 \pi i x u} \mathrm{~d} u$, we have $r(n)=\int_{0}^{1} g(u) \mathrm{e}^{-2 \pi i n u} \mathrm{~d} u$.

Note that

$$
\begin{aligned}
\frac{\mathrm{d}}{\mathrm{d} x} r(x) & =(-2 \pi i x) \int_{0}^{1} g(u) \mathrm{e}^{-2 \pi i x u} \mathrm{~d} u, \\
\frac{\mathrm{d}^{2}}{\mathrm{~d} x^{2}} r(x) & =(-2 \pi i x)^{2} \int_{0}^{1} g(u) \mathrm{e}^{-2 \pi i x u} \mathrm{~d} u,
\end{aligned}
$$

and so on.

Thus $r(x)$ is a smooth function. In particular, we may choose $g(u)=h_{y}(u)=h(u+i y)$ for a fixed $y>0$, where $h(z)=h(x+i y)$ is a cusp form under consideration as in the case of Jutila [3]. If $f$ is a smooth function on $[a, b]$, then $\phi(x)=f(x) \cdot r(x)$ is a smooth function on $[a, b]$ and we can apply Euler-Maclaurin or Poisson summation formula to the summation $\sum_{a<n \leq b} \phi(n)$.

We actually show that all the above results can be obtained using the two facts, namely,

(1) generalised Euler's summation formula (see Corollary 1 of Lemma 1),

(2) $\psi(x)=x-[x]-\frac{1}{2}=-\sum_{n=-\infty}^{\prime \infty} \mathrm{e}^{(2 \pi i n x / 2 \pi i n)}$, the series being convergent boundedly.

Next we prove the theorems. For this, we state our main results as lemmas and derive the proofs of our theorems from these lemmas.

Lemma 1. (Generalised Abel's summation formula). Let $(\lambda(n))_{n=-\infty}^{n=\infty}$ be a strictly increasing sequence of real numbers such that $\lambda(n) \rightarrow \infty$ as $n \rightarrow \infty$; and $\lambda(n) \rightarrow-\infty$ as $n \rightarrow-\infty$. Let $f$ be a function on an interval $[a, b]$ such that $f$ is continuous from left at 
every point of the sequence $(\lambda(n))$ with $a<\lambda(n) \leq b$. Let $(c(n))$ be a complex sequence and let $S(t)=\sum_{\lambda_{0}<\lambda(n) \leq t} c(n)$, where $\lambda_{0}$ is a fixed constant. Then

$$
\begin{aligned}
\sum_{a<\lambda(n) \leq b} c(n) f(\lambda(n)) & =\int_{a}^{b} f(t) \mathrm{d} S(t) \\
& =f(b) S(b)-f(a) S(a)-\int_{a}^{b} S(t) \mathrm{d} f(t) .
\end{aligned}
$$

A corresponding result may be given, if $f$ is continuous from right at every point $\lambda(n)$ with $a<\lambda(n) \leq b$.

\section{COROLLARY 1. (Generalised Euler's summation formula)}

Let $f$ be Riemann-integrable on the interval $[a, b]$ such that $f$ is continuous from left at every integer $n$ with $a<n \leq b$. Then

$$
\begin{aligned}
\sum_{a<n \leq b} f(n)= & \int_{a}^{b} f(u) \mathrm{d} u+\int_{a}^{b}\left(u-[u]-\frac{1}{2}\right) \mathrm{d} f(u) \\
& +f(a)\left(a-[a]-\frac{1}{2}\right)-f(b)\left(b-[b]-\frac{1}{2}\right) .
\end{aligned}
$$

Note. If $f$ is such that its derivative $f^{\prime}$ exists and is finite on $[a, b]$ and is integrable on $[a, b]$ (either in Riemann or Lebesgue sense), then we can replace $\int_{a}^{b}\left(u-[u]-\frac{1}{2}\right) \mathrm{d} f(u)$ by $\int_{a}^{b}\left(u-[u]-\frac{1}{2}\right) f^{\prime}(u) \mathrm{d} u$.

\section{COROLLARY 2. (Euler's summation formula for two variables)}

Let $f(x, y)$ be a function of two variables such that its partial derivatives up to second order are continuous in the rectangle $(a \leq x \leq b, c \leq y \leq d)$, where $a, b, c, d$ are integers. Then with obvious notations,

$$
\begin{aligned}
\sum_{c<n \leq d} \sum_{a<m \leq b} f(m, n)= & \int_{a}^{b} \int_{c}^{d} f(x, y) \mathrm{d} x \mathrm{~d} y \\
& +\int_{a}^{b} \int_{c}^{d} f_{x}(x, y)(x-[x]) \mathrm{d} x \mathrm{~d} y \\
& +\int_{a}^{b} \int_{c}^{d} f_{y}(x, y)(y-[y]) \mathrm{d} x \mathrm{~d} y \\
& +\int_{a}^{b} \int_{c}^{d} f_{x y}(x, y)(x-[x])(y-[y]) \mathrm{d} x \mathrm{~d} y .
\end{aligned}
$$

Proof. Using Euler's summation formula (for fixed $n$ ), we have

$$
\sum_{a<m \leq b} f(m, n)=\int_{a}^{b} f(x, n) \mathrm{d} x+\int_{a}^{b} f_{x}(x, n)(x-[x]) \mathrm{d} x .
$$

Hence,

$$
\begin{aligned}
\sum_{c<n \leq d}\left(\sum_{a<m \leq b} f(m, n)\right)= & \int_{a}^{b} \mathrm{~d} x\left(\sum_{c<n \leq d} f(x, n)\right) \\
& +\int_{a}^{b} \mathrm{~d} x(x-[x])\left(\sum_{c<n \leq d} f_{x}(x, n)\right) .
\end{aligned}
$$


Using Euler's summation formula once more, for the summations $\sum_{c<n \leq d} f(x, n)$ and $\sum_{c<n \leq d} f_{x}(x, n)$, we get the result as stated.

\section{COROLLARY 3.}

We have for integers $r, k$ with $0 \leq r<k$, if $f$ is continuous from left at the integer $n \equiv$ $r(\bmod k)$ with $a<n \leq b$, then

(I)

$$
\begin{aligned}
\sum_{\substack{a<n \leq b \\
n \equiv r(\bmod k)}} f(n)= & \frac{1}{k} \int_{a}^{b} f(u) \mathrm{d} u+\int_{a}^{b} \psi\left(\frac{u-r}{k}\right) \mathrm{d} f(u) \\
& +f(a) \cdot \psi\left(\frac{a-r}{k}\right)-f(b) \cdot \psi\left(\frac{b-r}{k}\right),
\end{aligned}
$$

provided $f$ is Riemann-integrable on $[a, b]$.

(II) Putting $r=0$ we get for $m \geq 1$, if $f$ is continuous on $[a, b]$

$$
\begin{aligned}
\sum_{a / m<n \leq b / m} f(m n)= & \frac{1}{m} \int_{a}^{b} f(u) \mathrm{d} u+\int_{a}^{b} \psi\left(\frac{u}{m}\right) \mathrm{d} f(u)+f(a) \psi\left(\frac{a}{m}\right) \\
& -f(b) \psi\left(\frac{b}{m}\right) .
\end{aligned}
$$

(III) We have for integer $m \geq 1$ and integer $k \geq 1$,

$$
\begin{aligned}
\sum_{\substack{a / m<n \leq b / m \\
n \equiv r(\bmod k)}} f(m n)= & \frac{1}{k m} \int_{a}^{b} f(u) \mathrm{d} u+\int_{a}^{b} \psi\left(\frac{(u / m)-r}{k}\right) \mathrm{d} f(u) \\
& +f(a) \psi\left(\frac{(a / m)-r}{k}\right)-f(b) \psi\left(\frac{(b / m)-r}{k}\right),
\end{aligned}
$$

provided $f$ is continuous on $[a, b]$.

Proofs of Theorems 1 and 2. Firstly, we shall find the Poisson and the Euler-Maclaurin analogues of $\sum_{a<n \leq b} \chi(n) f(n)$. We have

$$
\begin{aligned}
\sum_{a<n \leq b} \chi(n) f(n)= & \sum_{l=1}^{k} \chi(l)\left(\sum_{n \equiv l(\bmod k)} f(n)\right) \\
= & \sum_{l=1}^{k} \chi(l)\left\{\frac{1}{k} \int_{a}^{b} f(u) \mathrm{d} u+\int_{a}^{b} \psi\left(\frac{u-l}{k}\right) \mathrm{d} f(u)\right. \\
& \left.+f(a) \psi\left(\frac{a-l}{k}\right)-f(b) \psi\left(\frac{b-l}{k}\right)\right\}
\end{aligned}
$$


First, we shall obtain Poisson analogue. Integrating by parts, we have

$$
\begin{aligned}
\int_{a}^{b} \psi\left(\frac{u-l}{k}\right) \mathrm{d} f(u)= & -\int_{a}^{b}\left(\sum_{n=-\infty}^{\infty} \frac{\mathrm{e}^{2 \pi i n((u-l) / k)}}{2 \pi i n}\right) \mathrm{d} f(u) \\
= & -\sum_{n=-\infty}^{\infty} \frac{1}{2 \pi i n} \int_{a}^{b} \mathrm{e}^{2 \pi i n((u-l) / k)} \mathrm{d} f(u) \\
= & -\sum_{n}^{\prime} \frac{1}{2 \pi i n}\left\{\left[\mathrm{e}^{2 \pi i n((u-l) / k)} f(u)\right]_{u=a}^{u=b}\right. \\
& \left.-\frac{2 \pi i n}{k} \int_{a}^{b} f(u) \mathrm{e}^{2 \pi i n((u-l) / k)} \mathrm{d} u\right\} \\
= & -\sum_{n}^{\prime} \frac{1}{2 \pi i n}\left(\mathrm{e}^{2 \pi i n((b-l) / k)} f(b)-\mathrm{e}^{2 \pi i n((a-l) / k)} f(a)\right) \\
& +\frac{1}{k} \sum_{n}^{\prime} \int_{a}^{b} f(u) \mathrm{e}^{2 \pi i n((u-l) / k)} \mathrm{d} u .
\end{aligned}
$$

Thus Theorem 2(I) follows.

Next, we prove the Euler-Maclaurin analogue of $\sum_{a<n \leq b} \chi(n) f(n)$. Integrating by parts, we have

$$
\begin{aligned}
\int_{a}^{b} \psi\left(\frac{u-l}{k}\right) \mathrm{d} f(u)= & \int_{a}^{b} \psi\left(\frac{u-l}{k}\right) f^{\prime}(u) \mathrm{d} u \\
= & k \int_{a}^{b}(\mathrm{~d} / \mathrm{d} u) \psi_{1}\left(\frac{u-l}{k}\right) f^{\prime}(u) \mathrm{d} u \\
= & k\left[\psi_{1}\left(\frac{u-l}{k}\right) f^{\prime}(u)\right]_{u=a}^{b}-k \int_{a}^{b} \psi_{1}\left(\frac{u-l}{k}\right) f^{\prime \prime}(u) \mathrm{d} u \\
= & k\left(\psi_{1}\left(\frac{b-l}{k}\right) f^{\prime}(b)-\psi_{1}\left(\frac{u-l}{k}\right) f^{\prime}(a)\right) \\
& -k \int_{a}^{b} \psi_{1}\left(\frac{u-l}{k}\right) f^{\prime \prime}(u) \mathrm{d} u \\
= & k\left(\psi_{1}\left(\frac{b-l}{k}\right) f^{\prime}(b)-\psi_{1}\left(\frac{a-l}{k}\right) f^{\prime}(a)\right) \\
& -k^{2} \int_{a}^{b} \frac{\mathrm{d}}{\mathrm{d} u} \psi_{2}\left(\frac{u-l}{k}\right) f^{\prime \prime}(u) \mathrm{d} u,
\end{aligned}
$$

and so on. Thus, we get

$$
\begin{aligned}
\sum_{a<n \leq b} \chi(n) f(n)= & \sum_{l=1}^{k} \chi(l)\left\{\frac{1}{k} \int_{a}^{b} f(u) \mathrm{d} u\right. \\
& +\sum_{r=0}^{R}(-1)^{r+1} k^{r}\left(\psi_{r}\left(\frac{b-l}{k}\right) f^{(r)}(b)-\psi_{r}\left(\frac{a-l}{k}\right) f^{(r)}(a)\right) \\
& \left.-k^{R} \int_{a}^{b} \psi_{R}\left(\frac{u-l}{k}\right) f^{(R+1)}(u) \mathrm{d} u\right\} .
\end{aligned}
$$

This gives Theorem 1(I). Next, we deal with $\sum_{a<n \leq b} \mathrm{~d}(n) f(n)$. 
Now $\sum_{a<r \leq b} d(r) f(r)=\sum_{a<m n \leq b} f(m n)$.

Noting that the summation $a<m n \leq b$ means summation over lattice points between rectangular hyperbolae $x y=b$ and $x y=a$, the upper hyperbola included and the lower hyperbola excluded, we get

$$
\begin{aligned}
\sum_{a<r \leq b} d(r) f(r)= & \sum_{m \leq b}\left(\sum_{a / m<n \leq b / m} f(m n)\right) \\
= & \sum_{m \leq b} \frac{1}{m}\left(\int_{a}^{b} f(u) \mathrm{d} u\right)+\sum_{m \leq b}\left(\int_{a}^{b} \psi\left(\frac{u}{m}\right) \mathrm{d} f(u)\right. \\
& \left.+f(a) \psi\left(\frac{a}{m}\right)-f(b) \psi\left(\frac{b}{m}\right)\right) .
\end{aligned}
$$

Substituting the series for $\psi(u / m)$, we can obtain both the Poisson and the EulerMaclaurin analogues for $\sum_{a<n \leq b} d(n) f(n)$. Next we deal with $\sum_{a<n \leq b} d(n) \chi(n) f(n)$. Now

$$
\begin{aligned}
\sum_{a<n \leq b} d(n) \chi(n) f(n)= & \sum_{a<m n \leq b} \chi(m n) f(m n) \\
= & \sum_{m \leq b} \sum_{r_{2}=1}^{k} \chi\left(m r_{2}\right)\left(\sum_{\substack{a<m n \leq b \\
n \equiv r_{2}(\bmod k)}} f(m n)\right) \\
= & \sum_{m \leq b} \sum_{r_{2}=1}^{k} \chi\left(m r_{2}\right)\left(\sum_{\substack{a / m<n \leq b / m \\
n \equiv r_{2}(\bmod k)}} f(m n)\right) \\
= & \sum_{m \leq b} \sum_{r_{2}=1}^{k} \chi\left(m r_{2}\right)\left\{\frac{1}{k m} \int_{a}^{b} f(u) \mathrm{d} u\right. \\
& +\int_{a}^{b} \psi\left(\frac{(u / m)-r_{2}}{k}\right) \mathrm{d} f(u) \\
& +f(a) \psi\left(\frac{(a / m)-r_{2}}{k}\right) \\
& \left.-f(b) \psi\left(\frac{(b / m)-r_{2}}{k}\right)\right\} .
\end{aligned}
$$

Substituting the series for $\psi$, then interchanging $\sum$ and $\int$ and then integrating by parts in one way or the other as the case may be, we can get the Euler-Maclaurin or the Poisson analogue of $\sum_{a<n \leq b} d(n) \chi(n) f(n)$.

\section{References}

[1] Berndt B C, Character analogues of the Poisson and Euler-Maclaurin summation formulas with application, J. Number Theory 7 (1975) 413-445.

[2] Berndt B C and Schoenfeld Lowell, Periodic analogues of the Euler-Maclaurin and Poisson summation formulas with applications to number theory, Acta Arithmetica XXVIII (1975) 23-67 
[3] Jutila M, A method in the theory of exponential sums (Tata Institute of Fundamental Research, Mumbai) (1987)

[4] Voronoi M G, Sur une fonction transcendante et sis applications à la sommation de quelques séries, Ann. de l'Ecole Norm. Sup. (3) 21 (1904) 207-267, 459-533 